\title{
Derivative and Lipschitz Type Characterizations of Variable Exponent Bergman Spaces
}

\author{
Rumeng $\mathrm{Ma}^{1}$ and Jingshi $\mathrm{Xu}$ (D) $^{1,2}$ \\ ${ }^{1}$ School of Mathematics and Statistics, Hainan Normal University, Haikou 571158, China \\ ${ }^{2}$ School of Science, Guangxi University for Nationalities, Nanning 530006, China \\ Correspondence should be addressed to Jingshi Xu; jingshixu@126.com
}

Received 5 April 2018; Revised 20 June 2018; Accepted 16 July 2018; Published 1 August 2018

Academic Editor: Alberto Fiorenza

Copyright (c) 2018 Rumeng Ma and Jingshi Xu. This is an open access article distributed under the Creative Commons Attribution License, which permits unrestricted use, distribution, and reproduction in any medium, provided the original work is properly cited.

We give derivative and Lipschitz type characterizations of Bergman spaces with log-Hölder continuous variable exponent.

\section{Introduction}

The Bergman spaces were introduced in [1]. Since then, the theory of Bergman spaces has grown quickly, due to its connection with harmonic analysis, approximation theory, hyperbolic geometry, potential theory, and partial differential equations; see [2-5]. In particular, they can be characterized by derivatives and Lipschitz type conditions. Indeed, Zhu in [5] gave the derivatives characterizations of Bergman spaces. Wulan and Zhu in [6] gave Lipschitz type characterizations for Bergman spaces. We remark here that Lipschitz type characterizations for Sobolev spaces were considered in [7-11].

Recently, in [12], Chacón and Rafeiro introduced variable exponent Bergman spaces on the open unit ball of the plane and obtained that the Bergman projection and the Berezin transform are bounded and polynomials are dense in these spaces. Then in [13], Chacón, Rafeiro, and Vallejo gave a characterization of Carleson measures for variable exponent Bergman spaces. These results are generalizations of constant exponent Bergman spaces. The theory of variable function spaces has attracted many authors' attention for four decades. Since there are huge literatures, we only recommend [1416]. Motivated by those papers, in this paper, we shall extend the derivatives characterizations in [5] and Lipschitz type characterizations in [6] to variable exponent Bergman spaces on the open unit ball of $\mathbb{C}^{n}$ for any integer $n$. To state our results, we firstly recall some definitions.
We denote the Euclidean norm on $\mathbb{C}^{n}$ by $|\cdot|$. Then we let $\mathbb{B}^{n}=\left\{z \in \mathbb{C}^{n}:|z|<1\right\}$, the open unit ball in $\mathbb{C}^{n}$. Let $\mathrm{d} v$ be the normalized volume measure on $\mathbb{B}^{n}$. For any $\alpha>-1$, let $\mathrm{d} v_{\alpha}(z)=C_{\alpha}\left(1-|z|^{2}\right)^{\alpha} \mathrm{d} v(z)$, where $C_{\alpha}$ is a positive constant such that $v_{\alpha}\left(\mathbb{B}^{n}\right)=1$. In this paper, we only consider the case of $\alpha=0$.

For a measurable function $p: \mathbb{B}^{n} \longrightarrow[1, \infty)$, we call it a variable exponent and denote $p^{+}:=\operatorname{ess~sup}_{z \in \mathbb{B}^{n}} p(z), p^{-}:=$ ess $\inf _{z \in \mathbb{B}^{n}} p(z)$. Denote by $\mathscr{P}\left(\mathbb{B}^{n}\right)$ the set of all variable exponents with $p^{+}<\infty$. Let $p \in \mathscr{P}\left(\mathbb{B}^{n}\right)$. For a complexvalued measurable function $f$ on $\mathbb{B}^{n}$, we define the modular of $f$ by

$$
\rho_{p(\cdot)}(f):=\int_{\mathbb{B}^{n}}|f(z)|^{p(z)} \mathrm{d} \nu(z),
$$

and the Luxemburg-Nakano norm by

$$
\|f\|_{L^{p^{(\cdot)}\left(\mathbb{B}^{n}\right)}}:=\inf \left\{\lambda>0: \rho_{p(\cdot)}\left(\frac{f}{\lambda}\right) \leq 1\right\} .
$$

The variable Lebesgue space $L^{p(\cdot)}\left(\mathbb{B}^{n}, \mathrm{~d} \nu\right)$ is the set of all complex-valued measurable functions $f$ on $\mathbb{B}^{n}$ such that $\rho_{p(\cdot)}(f)<\infty$. It is a Banach space equipped with the Luxemburg-Nakano norm.

If $p \in \mathscr{P}\left(\mathbb{B}^{n}\right)$, then the variable exponent Bergman space $A^{p(\cdot)}\left(\mathbb{B}^{n}\right)$ is the class of all holomorphic functions on 
$\mathbb{B}^{n}$ which belong to the variable exponent Lebesgue space $L^{p(\cdot)}\left(\mathbb{B}^{n}, \mathrm{~d} \nu\right)$. It is easy to show that $A^{p(\cdot)}\left(\mathbb{B}^{n}\right)$ is a closed subspace of $L^{p(\cdot)}\left(\mathbb{B}^{n}, \mathrm{~d} \nu\right)$. When $p$ is a constant, these spaces are called weighted Bergman space with standard weights; see $[3,4]$ for details. As usual, we denote by $H\left(\mathbb{B}^{n}\right)$ the space of holomorphic functions on $\mathbb{B}^{n}$. by

Given $f \in H\left(\mathbb{B}^{n}\right)$, the radial derivative of $f$ at $z$ is defined

$$
\mathscr{R} f(z):=\sum_{k=1}^{n} z_{k} \frac{\partial f}{\partial z_{k}}(z) .
$$

The complex gradient of $f$ at $z$ is defined by

$$
|\nabla f(z)|:=\left[\sum_{k=1}^{n}\left|\frac{\partial f}{\partial z_{k}}(z)\right|^{2}\right]^{1 / 2}
$$

And the invariant complex gradient of $f$ at $z$ is given by

$$
|\widetilde{\nabla} f(z)|:=\left|\nabla\left(f \circ \varphi_{z}\right)(0)\right|,
$$

where $\varphi_{z}$ is the automorphism of $\mathbb{B}^{n}$ mapping 0 to $z$.

For any $a \in \mathbb{B}^{n}$, let $\varphi_{a}$ be a biholomorphic map on $\mathbb{B}^{n}$ such that $\varphi_{a}(0)=a$ and $\varphi_{a}^{-1}=\varphi_{a}$. The explicit formulas are available for $\varphi_{a}$ (see [5]).

Let $\beta$ be the Bergman metric on $\mathbb{B}^{n}$, namely, for $z, w \in \mathbb{B}^{\mathrm{n}}$,

$$
\beta(z, w):=\frac{1}{2} \log \frac{1+\left|\varphi_{z}(w)\right|}{1-\left|\varphi_{z}(w)\right|} .
$$

It was known that $\rho(z, w):=\left|\varphi_{z}(w)\right|$ is also a distance function on $\mathbb{B}^{n} . \rho$ is called the pseudohyperbolic metric on $\mathbb{B}^{n}$. For any $r \in(0,1)$ and $z \in \mathbb{B}^{n}$, we let $D(z, r):=\{w \in$ $\left.\mathbb{B}^{n}: \rho(w, z)<r\right\}$, the pseudohyperbolic ball centered at $z$ with radius $r . D(z, r)$ is Euclidean ball $B(x, a)=\left\{w \in \mathbb{B}^{n}\right.$ : $|w-x|<a\}$ with

$$
\begin{aligned}
& x=\frac{1-r^{2}}{1-r^{2}|z|^{2}} z, \\
& a=\frac{1-|z|^{2}}{1-r^{2}|z|^{2}} r .
\end{aligned}
$$

In particular, if $r$ is fixed, then the volume of $D(z, r)$ is comparable to $\left(1-|z|^{2}\right)^{n+1}$.

For any $R>0$ and $z \in \mathbb{B}^{n}$, we let $E(z, R):=\left\{w \in \mathbb{B}^{n}\right.$ : $\beta(w, z)<R\}$, the hyperbolic ball centered at $z$ with radius $R$. If $r \in(0,1)$, then $D(z, r)=E(z, R)$ with

$$
R=\frac{1}{2} \log \frac{1-r}{1+r} .
$$

Consequently, if $R$ is fixed, then the volume of $E(z, R)$ is also comparable to $\left(1-|z|^{2}\right)^{n+1}$.

Definition 1. A function $p: \mathbb{B}^{n} \longrightarrow \mathbb{R}$ is said to be log-Hölder continuous or satisfy the Dini-Lipschitz condition on $\mathbb{B}^{n}$ if there exists a positive constant $C_{\log }$ such that

$$
|p(z)-p(w)| \leq \frac{C_{\log }}{\log (1 /|z-w|)},
$$

for all $z, w \in \mathbb{B}^{n}$ such that $|z-w|<1 / 2$. We will denote by $\mathscr{P}^{\log }\left(\mathbb{B}^{n}\right)$ the set of all log-Hölder continuous functions on $\mathbb{B}^{n}$.

Now, the main result of the paper is the following.

Theorem 2. Suppose $p \in \mathscr{P}^{\log }\left(\mathbb{B}^{n}\right)$, and $f$ is holomorphic in $\mathbb{B}^{n}$. Then the following conditions are equivalent.
(a) $f \in A^{p(\cdot)}\left(\mathbb{B}^{n}\right)$.
(b) $|\widetilde{\nabla} f(\cdot)| \in L^{p(\cdot)}\left(\mathbb{B}^{n}, \mathrm{~d} v\right)$.
(c) $\left(1-|\cdot|^{2}\right)|\nabla f(\cdot)| \in L^{p(\cdot)}\left(\mathbb{B}^{n}, \mathrm{~d} v\right)$.
(d) $\left(1-|\cdot|^{2}\right)|\mathscr{R} f(\cdot)| \in L^{p(\cdot)}\left(\mathbb{B}^{n}, \mathrm{~d} v\right)$.
(e) There exists a continuous function $g$ in $L^{p(\cdot)}\left(\mathbb{B}^{n}, \mathrm{~d} \nu\right)$ such that, for all $z, w \in \mathbb{B}^{n}$,

$$
|f(z)-f(w)| \leq \rho(z, w)(g(z)+g(w)) .
$$

(f) There exists a continuous function $g$ in $L^{p(\cdot)}\left(\mathbb{B}^{n}, \mathrm{~d} \nu\right)$ such that, for all $z, w \in \mathbb{B}^{n}$,

$$
|f(z)-f(w)| \leq \beta(z, w)(g(z)+g(w)) .
$$

$(g)$ There exists a continuous function $g$ such that $\left(1-|\cdot|^{2}\right) g$ in $L^{p(\cdot)}\left(\mathbb{B}^{n}, \mathrm{~d} \nu\right)$ and for all $z, w \in \mathbb{B}^{n}$,

$$
|f(z)-f(w)| \leq|z-w|(g(z)+g(w)) .
$$

In Section 2, we shall collect some results which we shall need in the paper. The proof of Theorem 2 will be given in Section 3. Finally, we claim that the notation $a \leqslant b$ means there exists a constant $C>0$ such that $a \leq C b$, and $a \approx b$ means $a \leqslant b$ and $b \leqslant a$.

\section{Preliminaries}

In this section, we recall some preliminary results that we shall need in our paper.

Lemma 3 ([5], Lemma 2.20). Let $r$ be a positive number. Then there exists a positive constant $C_{r}$ such that

$$
\begin{aligned}
& C_{r}^{-1} \leq \frac{1-|z|^{2}}{|1-\langle z, w\rangle|} \leq C_{r}, \\
& C_{r}^{-1} \leq \frac{1-|z|^{2}}{1-|w|^{2}} \leq C_{r},
\end{aligned}
$$

for all $z, w \in \mathbb{B}^{n}$ with $\beta(z, w)<r$. Moreover, if $r$ is bounded above, then we may choose $C_{r}$ to be independent of $r$.

The following Jensen type inequality was proved in [17] in the context of spaces of homogeneous type (SHT).

Lemma 4. Suppose that $p(\cdot) \in \mathscr{P}^{\log }\left(\mathbb{B}^{n}\right)$. Then

$$
\begin{aligned}
& \left(f_{D(z, r)}|f(w)| \mathrm{d} v(w)\right)^{p(y)} \\
& \quad \leq\left(f_{D(z, r)}|f(w)|^{p(w)} \mathrm{d} v(w)+1\right),
\end{aligned}
$$

for all $y \in D(z, r), z \in \mathbb{B}^{n}$, provided that $\|f\|_{L^{p(\cdot)}\left(\mathbb{B}^{n}\right)} \leq 1$. 
Remark 5. Usually, Lemma 4 holds for Euclidean balls. We shall use it in pseudohyperbolic metric. Since each pseudohyperbolic ball $D(z, r)$ is actually Euclidean ball, we have the above form. And $f_{D(z, r)}|f(w)| \mathrm{d} \nu(w)=(1 / \mid D(z$, r)|) $\int_{D(z, r)}|f(w)| \mathrm{d} \nu(w)$.

Definition 6. Given a function $f \in L_{l o c}^{1}\left(\mathbb{B}^{n}\right)$, the HardyLittlewood maximal function of $f$, denoted by $M f$, is defined for any $z \in \mathbb{C}^{n}$ by

$$
M f(z):=\sup _{r>0} \frac{1}{B(z, r)} \int_{B(z, r)}|f(w)| \mathrm{d} w .
$$

Lemma 7 ([14], Theorem 3.16). Let $p \in \mathscr{P}^{\log }\left(\mathbb{B}^{n}\right)$. Then the Hardy-Littlewood maximal function is bounded in $L^{p(\cdot)}\left(\mathbb{B}^{n}\right)$, and it means that there exists a positive constant $C$ such that for each $f \in L^{p(\cdot)}\left(\mathbb{R}^{n}\right)$

$$
\|M f\|_{L^{p(\cdot)}\left(\mathbb{B}^{n}\right)} \leq C\|f\|_{L^{p(\cdot)}\left(\mathbb{B}^{n}\right)} .
$$

Let $\mathscr{F}$ denote a family of pairs of nonnegative measurable function and $A_{1}$ denote the Muckenhoupt $A_{1}$ weight.

Lemma 8 ([14], Theorem 5.24). Suppose that for some $p_{0} \geq 1$ the family $\mathscr{F}$ is such that, for all $w \in A_{1}$,

$$
\int_{\mathbb{B}^{n}} F(z)^{p_{0}} w(z) \mathrm{d} z \leq C_{0} \int_{\mathbb{B}^{n}} G(z)^{p_{0}} w(z) \mathrm{d} z,
$$

$$
(F, G) \in \mathscr{F} \text {. }
$$

Given $p(\cdot) \in \mathscr{P}\left(\mathbb{B}^{n}\right)$, if $p_{0} \leq p_{-} \leq p_{+}<\infty$ and the maximal operator is bounded on $L^{\left(p(\cdot) / p_{0}\right)^{\prime}}\left(\mathbb{B}^{n}\right)$, then there is a positive constant $C$ independent of $(F, G)$ such that

$$
\|F\|_{L^{p(\cdot)}\left(\mathbb{B}^{n}\right)} \leq C\|G\|_{L^{p(\cdot)}\left(\mathbb{B}^{n}\right)} .
$$

For $z \in \mathbb{C}^{n}$, we define the following radial test function:

$$
\eta(z)= \begin{cases}C \exp \left(\frac{1}{|z|^{2}-1}\right), & \text { if }|z|<1, \\ 0, & \text { if }|z| \geq 1,\end{cases}
$$

where $C>0$ is the normalizing constant in the sense $\int_{\mathbb{B}^{n}} \eta(z) \mathrm{d} \nu(z)=1$. For $r \in[1 / 2,1)$, we define $\eta_{r}(z):=\left(4 r^{2} /\right.$ $\left.(1-r)^{2}\right) \eta(2 r z /(1-r))$. Notice that $\eta_{r}$ is a $C^{\infty}$ function supported on the set $((1-r) / 2 r) \overline{\mathbb{B}^{n}}$ (where $\rho \overline{\mathbb{B}^{n}}$ stands for the closed unit ball with radius $\rho$ ) and $\int_{\mathbb{B}^{n}} \eta_{r}(z) \mathrm{d} \nu(z)=1$.

Definition 9. Given a function $f \in A^{p(\cdot)}\left(\mathbb{B}^{n}\right)$, we will define its mollified dilation $f_{r}:((1+r) / 2 r) \mathbb{B}^{n} \longrightarrow \mathbb{C}$ as

$$
f_{r}(z):=\int_{\mathbb{B}^{n}} f(r w) \eta_{r}(z-w) \mathrm{d} \nu(w),
$$

where $\rho \mathbb{B}^{n}$ stands for the complex ball with radius $\rho$.

The following lemma is the counterpart of Theorem 3.5 in [12] for any integer $n$.
Lemma 10. Let $p(\cdot) \in \mathscr{P}^{\log }\left(\mathbb{B}^{n}\right)$ and $f \in A^{p(\cdot)}\left(\mathbb{B}^{n}\right)$. Then there exists a positive constant $C$ for $r \in(1 / 2,1), f_{r} \in A^{p(\cdot)}\left(\mathbb{B}^{n}\right)$ such that

$$
\sup _{1 / 2 \leq r<1}\left\|f_{r}\right\|_{A^{p(\cdot)}\left(\mathbb{B}^{n}\right)} \leq C\|f\|_{A^{p(\cdot)}\left(\mathbb{B}^{n}\right)}
$$

Moreover, $\left\|f_{r}-f\right\|_{A^{p(\cdot)}\left(\mathbb{B}^{n}\right)} \longrightarrow 0$ as $r \longrightarrow 1^{-}$.

Proof. Let $h \in A^{p^{\prime}(\cdot)}\left(\mathbb{B}^{n}\right),\|h\|_{A^{p^{\prime}(\cdot)\left(\mathbb{B}^{n}\right)}}=1,1 / p(x)+1 / p^{\prime}(x)=$ 1. Then by making change of variable and using Fubini's theorem, we obtain

$$
\begin{aligned}
& \left|\int_{\mathbb{B}^{n}} f_{r}(z) h(z) \mathrm{d} \nu(z)\right| \leq \int_{\mathbb{B}^{n}}|h(z)| \\
& \cdot \int_{B(z,(1-r) / 2 r)}|f(r w)| \eta_{r}(z-w) \mathrm{d} \nu(w) \mathrm{d} \nu(z) \\
& =\int_{B(0,(1-r) / 2 r)} \eta_{r}(w) \int_{\mathbb{B}^{n}}|f(r(z-w))||h(z)| \mathrm{d} \nu(z) \mathrm{d} \nu(w) \\
& =\frac{1}{r^{n}} \int_{B(0,(1-r) / 2 r)} \eta_{r}(w) \\
& \cdot \int_{B(-r w, r)}|f(z)|\left|h\left(\frac{z}{r}+w\right)\right| \mathrm{d} v(z) \mathrm{d} v(w)=\frac{1}{r^{n}} \\
& \cdot \int_{B(0,(1+r) / 2 r)}|f(z)| \\
& \cdot \int_{B(0,(1-r) / 2 r)} \chi_{B(-r w, r)}(z) \eta_{r}(w)|| h\left(\frac{z}{r}+w\right) \mid \mathrm{d} \nu(w) \mathrm{d} \nu(z) \\
& \leq \frac{1}{r^{n}} \int_{B(0,(1+r) / 2 r)}|f(z)| \\
& \cdot \int_{B(z / r,(1-r) / 2 r) \cap \mathbb{B}^{n}} \eta_{r}\left(w-\frac{z}{r}\right)|h(w)| \mathrm{d} \nu(w) \mathrm{d} \nu(z) \\
& \lesssim \int_{B(0,(1+r) / 2 r)}|f(z)| \\
& \cdot \frac{1}{|B(z / r,(1-r) / 2 r)|} \int_{B(z / r,(1-r) / 2 r)}|h(w)| \mathrm{d} \nu(w) \mathrm{d} \nu(z) .
\end{aligned}
$$

Since $B(z / r,(1-r) / 2 r) \subset B(z, 3(1-r) / 2 r)$, we have

$$
\begin{aligned}
& \frac{1}{|B(z / r,(1-r) / 2 r)|} \int_{B(z / r,(1-r) / 2 r)}|h(w)| \mathrm{d} \nu(w) \\
& \quad \leq \frac{9}{|B(z, 3(1-r) / 2 r)|} \int_{B(z, 3(1-r) / 2 r)}|h(w)| \mathrm{d} \nu(w) .
\end{aligned}
$$

Therefore, considering the maximal function $M h$, and using Hölder's inequality and Lemma 7 we have that

$$
\begin{aligned}
& \left|\int_{\mathbb{B}^{n}} f_{r}(z) h(z) \mathrm{d} \nu(z)\right| \\
& \quad \leq \int_{B(0,(1+r) / 2)}|f(z)| M h(z) \mathrm{d} \nu(z) \\
& \quad \leq\|f\|_{A^{p(\cdot)}\left(\mathbb{B}^{n}\right)}\|M h\|_{A^{p^{\prime}(\cdot)}\left(\mathbb{B}^{n}\right)} \\
& \quad \leq\|f\|_{A^{p(\cdot)}\left(\mathbb{B}^{n}\right)}\|h\|_{A^{p^{\prime} \cdot(\cdot)}\left(\mathbb{B}^{n}\right)}
\end{aligned}
$$

which shows the first part of the lemma. 
For the second part, fix $\varepsilon>0$ and choose a function $g$ with compact support on $\mathbb{B}^{n}$, such that $\|f-g\|_{A^{p(\cdot)}\left(\mathbb{B}^{n}\right)}<\varepsilon$ (see [14] Theorem 2.72). Then by the previous part of the proof,

$$
\begin{aligned}
\left\|f_{r}-f\right\|_{A^{p(\cdot)}\left(\mathbb{B}^{n}\right)} \leq & \left\|(f-g)_{r}\right\|_{L^{p(\cdot)}\left(\mathbb{B}^{n}\right)}+\left\|g_{r}-g\right\|_{L^{p(\cdot)}\left(\mathbb{B}^{n}\right)} \\
& +\|g-f\|_{L^{p(\cdot)}\left(\mathbb{B}^{n}\right)} \\
\leq & \varepsilon+\left\|g_{r}-g\right\|_{L^{p(\cdot)}\left(\mathbb{B}^{n}\right)} .
\end{aligned}
$$

Therefore, we only need to prove the convergence in norm for the compactly supported function $g$. Defining $G(z):=$ $g(z) /\left(2\|g\|_{L^{\infty}}\left(\mathbb{B}^{n}\right)\right)$, we obtain that $\|G\|_{L^{\infty}}\left(\mathbb{B}^{n}\right) \leq 1 / 2$. And

$$
\begin{aligned}
\left|G_{r}(z)\right| & \leq \int_{\mathbb{B}^{n}}|G(r w)| \eta_{r}(z-w) \mathrm{d} v(w) \\
& \leq\|G\|_{L^{\infty}}\left(\mathbb{B}^{n}\right) \int_{\mathbb{B}^{n}} \eta_{r}(z-w) \mathrm{d} v(w) \leq \frac{1}{2} .
\end{aligned}
$$

Thus, $\left\|G_{r}-g\right\|_{L^{\infty}}\left(\mathbb{B}^{n}\right) \leq 1$ and consequently

$$
\begin{aligned}
& \int_{\mathbb{B}^{n}}\left|g_{r}(z)-g(z)\right|^{p(z)} \mathrm{d} v(z) \\
& \quad=\int_{\mathbb{B}^{n}}\left(2\|g\|_{L^{\infty}}\left(\mathbb{B}^{n}\right)\right)^{p(z)}\left|G_{r}(z)-g(z)\right|^{p(z)} \mathrm{d} v(z) \\
& \leq\left(2\|g\|_{L^{\infty}}\left(\mathbb{B}^{n}\right)+1\right)^{p^{+}} \int_{\mathbb{B}^{n}}\left|G_{r}(z)-g(z)\right|^{p^{-}} \mathrm{d} \nu(z) .
\end{aligned}
$$

Therefore, we have reduced the convergence to the case of a constant exponent.

In this case, from [3] we know that radial dilations $G(r \cdot)$ converge in $p^{-}$-norm to $G$. Consequently, if we define the translation operator $\tau_{z} f(w):=f(z-w)$, then by Minkowski's inequality

$$
\begin{aligned}
& \left\|G_{r}-G\right\|_{L^{p-\left(\mathbb{B}^{n}\right)}} \\
& \quad \leq \int_{\mathbb{B}^{n}}\left\|\tau_{z} G(r \cdot)-G\right\|_{L^{p-\left(\mathbb{B}^{n}\right)}} \eta_{r}(z) \mathrm{d} \nu(z) \\
& \quad \leq \int_{\mathbb{B}^{n}}\left\|\tau_{z} G(r \cdot)-G(r \cdot)\right\|_{L^{p-}\left(\mathbb{B}^{n}\right)} \eta_{r}(z) \mathrm{d} \nu(z) \\
& \quad+\|G(r \cdot)-G\|_{L^{p-\left(\mathbb{B}^{n}\right)}},
\end{aligned}
$$

and the result follows from the continuity of $\tau_{z}$ on the space $L^{p^{-}}\left(\mathbb{B}^{n}\right)$.

From the above lemma, we have the following lemma.

Lemma 11. Let $p(\cdot) \in \mathscr{P}^{\log }\left(\mathbb{B}^{n}\right)$. Then the set of holomorphic polynomials is dense in $A^{p(\cdot)}\left(\mathbb{B}^{n}\right)$.

Lemma 12 ([5], Proposition 1.13). If $\alpha$ is real and $f$ is in $L^{1}\left(\mathbb{B}^{n}, \mathrm{~d} v\right)$, then

$$
\begin{aligned}
& \int_{\mathbb{B}^{n}} f \circ \varphi(w) \mathrm{d} v_{\alpha}(w) \\
& \quad=\int_{\mathbb{B}^{n}} f(w) \cdot \frac{\left(1-|z|^{2}\right)^{n+1+\alpha}}{|1-\langle z, w\rangle|^{2(n+1+\alpha)}} \mathrm{d} v_{\alpha}(w),
\end{aligned}
$$

where $\varphi$ is any automorphism of $\mathbb{B}^{n}$ and $\varphi(0)=z$.
Lemma 13 ([5], Lemma 2.14). If $f$ is holomorphic in $\mathbb{B}^{n}$, then for all $z \in \mathbb{B}^{n}$

$$
\left(1-|z|^{2}\right)|\mathscr{R} f(z)| \leq\left(1-|z|^{2}\right)|\nabla f(z)| \leq|\widetilde{\nabla} f(z)| .
$$

Lemma 14 ([5], Theorem 1.12). Let $c \in \mathbb{R}$ and $t \in(-1, \infty)$. For $z \in \mathbb{B}^{n}$, define

$$
\begin{aligned}
& I_{c}(z):=\int_{\mathbb{S}^{n}} \frac{\mathrm{d} \sigma(\xi)}{|1-\langle z, \xi\rangle|^{n+c}}, \\
& J_{c, t}(z):=\int_{\mathbb{B}^{n}} \frac{\left(1-|w|^{2}\right)^{t} \mathrm{~d} \nu(w)}{|1-\langle z, w\rangle|^{n+1+t+c}} .
\end{aligned}
$$

When $c<0$, then $I_{c}$ and $J_{c, t}$ are bounded in $\mathbb{B}$. When $c>0$, then

$$
I_{c}(z) \sim\left(1-|z|^{2}\right)^{-c} \sim J_{c, t} .
$$

Finally,

$$
I_{0}(z) \sim \log \frac{1}{1-|z|^{2}} \sim J_{0, t}(z) .
$$

The notation $a(z) \sim b(z)$ means that the ratio $a(z) / b(z)$ has a positive finite limit as $|z| \longrightarrow 1$.

The Bergman projection operator $P$ is defined for functions $f$ on $\mathbb{B}^{n}$ by

$$
P(f)(z):=\int_{\mathbb{B}^{n}} \frac{f(\xi)}{(1-\langle z, \xi\rangle)^{n+1}} \mathrm{~d} \nu(\xi) .
$$

To proceed, we need the class $B_{p}$ for $p \in(1, \infty)$. Let $\omega$ be a positive measurable function, and $\omega$ is called belonging to the class $B_{p}$ if there exists a constant $C$ such that, for every pseudo-ball $B \subset \mathbb{B}^{n}$,

$$
\frac{\omega(B)}{\nu(B)}\left(\frac{1}{\nu(B)} \int_{B} \omega(z)^{-1 /(p-1)} \mathrm{d} \nu(z)\right)^{p-1} \leq C,
$$

where $\omega(B)=\int_{\mathbb{B}^{n}} \omega(z) \mathrm{d} \nu(z)$.

Lemma 15 ([18], Theorem 1). Let $\omega \in L^{1}\left(\mathbb{B}^{n}\right)$. A necessary and sufficient condition for the Bergman projection to be bounded on $L^{p}(\omega)$ is that $\omega$ belongs to the class $B_{p}$ for $p \in(1, \infty)$.

Lemma 16. Let $p \in \mathscr{P}^{\log }\left(\mathbb{B}^{n}\right)$. Then the Bergman projection operator $P$ is bounded from $L^{p(\cdot)}\left(\mathbb{B}^{n}\right)$ onto $A^{p(\cdot)}\left(\mathbb{B}^{n}\right)$.

Proof. We follow the proof of Theorem 4.4 in [12]. It is clear that $P f$ is holomorphic function on $\mathbb{B}^{n}$, so we only need to prove that $P$ is bounded and surjective from $L^{p(\cdot)}\left(\mathbb{B}^{n}\right)$ to $L^{p(\cdot)}\left(\mathbb{B}^{n}\right)$. By the property of the Muckenhoupt $A_{1}$ (see [19]), if $w \in A_{1}$ then $w \in A_{q}$ for $q>1$. Now we pick $p_{0}$ such that $1<p_{0}<p_{-}$. By the remark 1 in [18], we have that $w \in B_{p_{0}}$. By Lemma 15 we conclude that the family $\mathscr{F}=\left\{(P f, f): f^{f} \in\right.$ $\left.L^{p_{0}}\left(\mathbb{B}^{n}\right)\right\}$ satisfies (17). By the fact that $\left(p(\cdot) / p_{0}\right)^{\prime} \in \mathscr{P}^{\log }\left(\mathbb{B}^{n}\right)$ 
and Lemmas 7 and 8 , then there exists a constant $C>0$ such that

$$
\|P f\|_{L^{p^{(\cdot)}\left(\mathbb{B}^{n}\right)}} \leq C_{p(\cdot)}\|f\|_{L^{p^{(\cdot)}\left(\mathbb{B}^{n}\right)}} .
$$

This shows that $P$ is bounded in $L^{p(\cdot)}\left(\mathbb{B}^{n}\right)$.

In order to prove that $P$ is surjective, we use the fact that $P g=g$ for every $g \in A^{2}\left(\mathbb{B}^{n}\right)$. In particular, this equality holds for any polynomial. Thus, if $f \in A^{p(\cdot)}\left(\mathbb{B}^{n}\right)$, we use Lemma 11 to find a sequence of polynomials $q_{m}$ converging in $A^{p(\cdot)}\left(\mathbb{B}^{n}\right)$ to $f$. But since $q_{m}=P q_{m} \longrightarrow P f$ in $A^{p(\cdot)}\left(\mathbb{B}^{n}\right)$ then we have that $P f=f$.

Lemma 17 ([5], Lemma 2.24). Suppose $r>0, q>0$, and $\alpha>-1$. Then there exists a constant $C>0$ such that

$$
|f(z)|^{q} \leq \frac{C}{\left(1-|z|^{2}\right)^{n+1+\alpha}} \int_{D(z, r)}|f(w)|^{q} \mathrm{~d} v_{\alpha}(w),
$$

for all $f \in H\left(\mathbb{B}^{n}\right)$ and all $z \in \mathbb{B}^{n}$.

The last result deals with the limits of $\beta(z, w)$ and $\rho(z, w)$ as $w$ tends $z$ in the radial direction.

Lemma 18 ([6], Lemma 5.2). Suppose $z \in \mathbb{B}^{n}$ and $w=t z$, where $t$ is a scalar. Then

$$
\lim _{w \longrightarrow z} \frac{\beta(z, w)}{|z-w|}=\lim _{w \longrightarrow z} \frac{\rho(z, w)}{|z-w|}=\frac{1}{1-|z|^{2}} .
$$

\section{Proof of Theorem 2}

Proof of Theorem 2. We shall divide the proof into 8 steps. In Step 1, by Lemma 13 we obtain that (b) implies (c) and (c) implies (d).

In Step 2, we prove (a) implies (b). We firstly consider that $f \in A^{p(\cdot)}\left(\mathbb{B}^{n}\right)$ such that $\|f\|_{L^{p(\cdot)}\left(\mathbb{B}^{n}\right)}=1$. We follow the idea of the proof of Theorem 2.16 in [5] and make some crucial modifications where needed. Fix $r \in(0,1)$. It follows from Lemma 2.4 in [5] that for fixed $\beta>\alpha=0$ there exists a constant $C>0$ such that

$$
|\nabla g(0)| \leq C \int_{D(z, r)}|g(w)| \mathrm{d} v_{\beta}(w),
$$

for all holomorphic $g$ in $\mathbb{B}^{n}$.

Now, for each $z \in \mathbb{B}^{n}$, let $\varphi_{z}$ be the biholomorphic mapping of $\mathbb{B}^{n}$ which interchanges 0 to $z$, and let $g=f \circ \varphi_{z}$. Then by making an obvious change of variables according to Lemma 12, we obtain

$$
\begin{aligned}
& |\widetilde{\nabla} f(z)| \leq C\left(1-|z|^{2}\right)^{n+1+\beta} \\
& \quad \cdot \int_{D(z, r)} \frac{|f(w)|}{|1-\langle z, w\rangle|^{2(n+1+\beta)}} \mathrm{d} v_{\beta}(w) \\
& \quad=C\left(1-|z|^{2}\right)^{n+1+\beta}
\end{aligned}
$$

$$
\begin{aligned}
& \cdot \int_{D(z, r)} \frac{|f(w)| C_{\beta}\left(1-|w|^{2}\right)^{\beta}}{|1-\langle z, w\rangle|^{2(n+1+\beta)}} \mathrm{d} \nu(w) \\
& \leq \frac{C}{\left(1-|z|^{2}\right)^{n+1}} \int_{D(z, r)}|f(w)| \mathrm{d} \nu(w) .
\end{aligned}
$$

Since the volume of $D(z, r)$ is comparable to $\left(1-|z|^{2}\right)^{n+1}$, then we have

$$
|\widetilde{\nabla} f(z)| \leq C f_{D(z, r)}|f(w)| \mathrm{d} v(w) .
$$

Then by Lemma 4 we have

$$
\begin{aligned}
& |\widetilde{\nabla} f(z)|^{p(z)} \leqslant f_{D(z, r)}|f(w)|^{p(w)} \mathrm{d} v(w)+1 \\
& \quad \leq \int_{D(z, r)} \frac{|f(w)|^{p(w)}\left(1-|z|^{2}\right)^{n+1+\beta}}{|1-\langle z, w\rangle|^{2(n+1+\beta)}} \mathrm{d} v_{\beta}(w)+1 .
\end{aligned}
$$

Integrating both sides of the above inequality over $\mathbb{B}^{n}$ with respect to $\mathrm{d} v(z)$ and using Fubini's theorem, we have that

$$
\begin{aligned}
& \int_{\mathbb{B}^{n}}|\widetilde{\nabla} f(z)|^{p(z)} \mathrm{d} v(z) \\
& \leq \int_{\mathbb{B}^{n}} \int_{D(z, r)} \frac{|f(w)|^{p(w)}\left(1-|z|^{2}\right)^{n+1+\beta}}{|1-\langle z, w\rangle|^{2(n+1+\beta)}} \mathrm{d} v_{\beta}(w) \mathrm{d} v(z)+1 \\
& \leq \int_{\mathbb{B}^{n}}|f(w)|^{p(w)} \mathrm{d} \gamma_{\beta}(w) \int_{\mathbb{B}^{n}} \frac{\left(1-|z|^{2}\right)^{n+1+\beta}}{|1-\langle z, w\rangle|^{2(n+1+\beta)} \mathrm{d} v(z)+1} \\
& \leq \int_{\mathbb{B}^{n}}|f(w)|^{p(w)}\left(1-|w|^{2}\right)^{\beta} \mathrm{d} v(w) \int_{\mathbb{B}^{n}} \frac{\left(1-|z|^{2}\right)^{n+1+\beta}}{|1-\langle z, w\rangle|^{2(n+1+\beta)}} \mathrm{d} v(z) \\
& \quad+1 \\
& \leq \int_{\mathbb{B}^{n}}|f(w)|^{p^{(w)}}\left(1-|w|^{2}\right)^{\beta} \mathrm{d} v(w) \int_{\mathbb{B}^{n}} \frac{\left(1-|z|^{2}\right)^{n+1+\beta}}{|1-\langle z, w\rangle|^{2(n+1+\beta)}} \mathrm{d} v(z) \\
& \quad+1 .
\end{aligned}
$$

By Lemma 14 we have that

$$
\int_{\mathbb{B}^{n}} \frac{\left(1-|z|^{2}\right)^{n+1+\beta}}{|1-\langle z, w\rangle|^{2(n+1+\beta)}} \mathrm{d} \nu(z) \sim\left(1-|w|^{2}\right)^{-\beta} .
$$

Thus using Lemma 3 and the above result we obtain that

$$
\begin{aligned}
& \int_{\mathbb{B}^{n}}|\widetilde{\nabla} f(z)|^{p(z)} \mathrm{d} v(z) \\
& \quad \leq \int_{\mathbb{B}^{n}}|f(w)|^{p(w)}\left(1-|w|^{2}\right)^{\beta}\left(1-|w|^{2}\right)^{-\beta} \mathrm{d} v(w) \\
& \quad+1 \lesssim \int_{\mathbb{B}^{n}}|f(w)|^{p(w)} \mathrm{d} v(w)+1 .
\end{aligned}
$$

Therefore $\widetilde{\nabla} f \in L^{p(\cdot)}\left(\mathbb{B}^{n}, \mathrm{~d} v\right)$. 
Finally, for a general $0 \neq f \in A^{p(\cdot)}\left(\mathbb{B}^{n}\right)$, define $h=$ $f\|f\|_{L^{p(\cdot)}\left(\mathbb{B}^{n}\right)}^{-1}$, and we apply the previous result for $h$ and conclude that $\widetilde{\nabla} f \in L^{p(\cdot)}\left(\mathbb{B}^{n}, \mathrm{~d} \nu\right)$.

In Step 3, to prove (d) implies (a), we assume that $f$ is a holomorphic function in $\mathbb{B}^{n}$ such that the function $(1-$ $\left.|z|^{2}\right)|\mathscr{R} f(z)| \in L^{p(\cdot)}\left(\mathbb{B}^{n}, \mathrm{~d} \nu\right)$. Let $\beta$ be a sufficiently large positive constant. Then by the proof of Theorem 2.16 in [5] we have that

$$
\begin{aligned}
|f(z)-f(0)| & \leq \int_{\mathbb{B}^{n}} \frac{|\mathscr{R} f(w)|}{|1-\langle z, w\rangle|^{n+\beta}} \mathrm{d} v_{\beta}(w) \\
& =C_{\beta} \int_{\mathbb{B}^{n}} \frac{|\mathscr{R} f(w)|\left(1-|w|^{2}\right)^{\beta}}{|1-\langle z, w\rangle|^{n+\beta}} \mathrm{d} \nu(w) \\
& \lesssim \int_{\mathbb{B}^{n}} \frac{|\mathscr{R} f(w)|\left(1-|w|^{2}\right)}{|1-\langle z, w\rangle|^{n+1}} \mathrm{~d} \nu(w) \\
& \lesssim P\left(\left(1-|\cdot|^{2}\right)|\mathscr{R} f(\cdot)|\right)(z),
\end{aligned}
$$

where $P$ is the Bergman projection operator. By Lemma 16 we know that $f(\cdot)-f(0) \in L^{p(\cdot)}\left(\mathbb{B}^{n}, d v\right)$. Therefore $f \in A^{p(\cdot)}\left(\mathbb{B}^{n}\right)$.

In Step 4, we prove (a) implies (e). If $f \in A^{p(\cdot)}\left(\mathbb{B}^{n}\right)$ and $z \in \mathbb{B}^{n}$, we have

$$
f(z)-f(0)=\int_{0}^{1}\left[\sum_{k=1}^{n} z_{k} \frac{\partial f}{\partial z_{k}}(t z)\right] \mathrm{d} t .
$$

Fix $r \in(0,1)$. It follows that, for $\rho(z, 0)<r$, we have

$$
\begin{aligned}
& |f(z)-f(0)|=\left|\int_{0}^{1}\left[\sum_{k=1}^{n} z_{k} \frac{\partial f}{\partial z_{k}}(t z)\right] \mathrm{d} t\right| \\
& \leq \int_{0}^{1}\left|\left[\sum_{k=1}^{n} z_{k} \frac{\partial f}{\partial z_{k}}(t z)\right]\right| \mathrm{d} t \\
& \quad \leq \int_{0}^{1}\left(\sum_{k=1}^{n} z_{k}^{2}\right)^{1 / 2}\left(\sum_{k=1}^{n}\left(\frac{\partial f}{\partial z_{k}}(t z)\right)^{2}\right)^{1 / 2} \mathrm{~d} t \\
& \quad \leq \int_{0}^{1}|z||\nabla f(t z)| \mathrm{d} t=|z| \int_{0}^{1}|\nabla f(t z)| \mathrm{d} t \\
& \quad \leq|z| \sup \{|\nabla f(w)|: w \in D(0, r)\} .
\end{aligned}
$$

Since in the relatively compact set $D(0, r)$ the Euclidean metric is comparable to the pseudohyperbolic metric, then $|\nabla f(w)|$ is comparable to $|\widetilde{\nabla} f(w)|$ in the relatively compact set $D(0, r)$. Thus, there is a constant $C>0$, which depends only on $r$, such that

$$
\begin{aligned}
& |f(z)-f(0)| \\
& \quad \leq C \rho(z, 0) \sup \{|\widetilde{\nabla} f(w)|: w \in D(0, r)\},
\end{aligned}
$$

for all $z \in D(0, r)$. Replacing $f$ by $f \circ \varphi_{w}$, and $z$ by $\varphi_{w}(z)$, respectively, and using the Möbius invariance of the pseudohyperbolic metric and the invariant gradient, we obtain

$$
\begin{aligned}
& |f(z)-f(w)| \\
& \quad \leq C \rho(z, w) \sup \{|\widetilde{\nabla} f(u)|: u \in D(z, r)\},
\end{aligned}
$$

for all $z$ and $w$ in $\mathbb{B}^{n}$ with $\rho(z, w)<r$.

Let

$$
g(z):=\frac{|f(z)|}{r}+h(z)
$$

where $h(z)=C \sup \{|\widetilde{\nabla} f(u)|: u \in D(z, r)\}$. Then it is easy to see that the function $g$ is continuous on $\mathbb{B}^{n}$ and (10) holds. Now we only need to prove that $g \in L^{p(\cdot)}\left(\mathbb{B}^{n}, \mathrm{~d} \nu\right)$. Since $f$ is already in $L^{p(\cdot)}\left(\mathbb{B}^{n}, \mathrm{~d} v\right)$, the remainder is to show that $h \in$ $L^{p(\cdot)}\left(\mathbb{B}^{n}, \mathrm{~d} v\right)$.

Recall that if $r$ and $R$ satisfy (8), then $D(z, r)=E(z, R)$. So we can choose $r^{\prime} \in(0,1)$ such that $D\left(z, r^{\prime}\right)=E(z, 2 R)$ for all $z \in \mathbb{D}$. For any $u \in E(z, R)$, any $w_{1} \in E(u, R)$, we have $\beta\left(w_{1}, u\right)<R$. Then we have $\beta(u, z)<R$ due to $u \in E(z, R)$. By the triangle inequality, we have

$$
\beta\left(w_{1}, z\right) \leq \beta\left(w_{1}, u\right)+\beta(u, z)<R+R<2 R .
$$

So $w_{1} \in E(z, 2 R)$. That is, $E(u, R) \subset E(z, 2 R)$. Equivalently, we have $D(u, r) \subset D\left(z, r^{\prime}\right)$ whenever $u \in D(z, r)$.

By Lemmas 17 and 3 we obtain that

$$
\begin{aligned}
& |h(z)| \lesssim \frac{1}{\left(1-|z|^{2}\right)^{n+1}} \int_{D(z, r)}|h(w)| \mathrm{d} v(w) \\
& \quad \lesssim \frac{1}{\left(1-|z|^{2}\right)^{n+1}} \\
& \quad \cdot \int_{D(z, r)}|C \sup \{|\widetilde{\nabla} f(u)|: u \in D(w, r)\}| \mathrm{d} v(w) \\
& \quad \lesssim \frac{1}{\left(1-|z|^{2}\right)^{n+1}} \int_{D\left(z, r^{\prime}\right)}|\widetilde{\nabla} f(w)| \mathrm{d} v(w) \\
& \quad \leqslant f_{D\left(z, r^{\prime}\right)}|\widetilde{\nabla} f(w)| \mathrm{d} v(w),
\end{aligned}
$$

where we used that the volume of $D\left(z, r^{\prime}\right)$ is comparable to $\left(1-|z|^{2}\right)^{n+1}$. Without loss of generality, we assume $\|\widetilde{\nabla} f\|_{L^{p(\cdot)}\left(\mathbb{B}^{n}, d v\right)}=1$. Therefore, using Lemma 4 we obtain that

$$
\begin{aligned}
|h(z)|^{p(z)} & \leqslant f_{D\left(z, r^{\prime}\right)}|\widetilde{\nabla} f(w)|^{p(w)} \mathrm{d} v(w)+1 \\
& \leqslant \frac{1}{\left(1-|z|^{2}\right)^{n+1}} \int_{D\left(z, r^{\prime}\right)}|\widetilde{\nabla} f(w)|^{p(w)} \mathrm{d} v(w) \\
& +1
\end{aligned}
$$


Integrating both sides of the above inequality over $\mathbb{B}^{n}$ with respect to $\mathrm{d} \nu(z)$ and using Fubini's theorem, we have that

$$
\begin{aligned}
& \int_{\mathbb{B}^{n}}|h(z)|^{p(z)} \mathrm{d} \nu(z) \\
& \leq \int_{\mathbb{B}^{n}} \frac{1}{\left(1-|z|^{2}\right)^{n+1}} \int_{D\left(z, r^{\prime}\right)}|\widetilde{\nabla} f(w)|^{p(w)} \mathrm{d} \nu(w) \mathrm{d} \nu(z) \\
& \quad+1 \\
& \leq \int_{\mathbb{B}^{n}}|\widetilde{\nabla} f(w)|^{p(w)} \mathrm{d} \nu(w) \int_{D\left(w, r^{\prime}\right)} \frac{1}{\left(1-|z|^{2}\right)^{n+1}} \mathrm{~d} \nu(z) \\
& \quad+1 \lesssim \int_{\mathbb{B}^{n}}|\widetilde{\nabla} f(w)|^{p(w)} \mathrm{d} \nu(w)+1 .
\end{aligned}
$$

Due to $f \in A^{p(\cdot)}\left(\mathbb{B}^{n}\right)$, by Step 2 we have that $|\widetilde{\nabla} f(\cdot)| \in$ $L^{p(\cdot)}\left(\mathbb{B}^{n}, \mathrm{~d} \nu\right)$. Thus $h \in L^{p(\cdot)}\left(\mathbb{B}^{n}, \mathrm{~d} \nu\right)$.

In Step 5, it is easy to see that (e) implies (f), according to $\rho(z, w) \leq \beta(z, w)$, for all $z, w \in \mathbb{B}^{n}$.

In Step 6, we prove that (f) implies (a). Assume that there exists a continuous function $g \in L^{p(\cdot)}\left(\mathbb{B}^{n}, \mathrm{~d} \nu\right)$ such that (11) holds for all $z, w \in \mathbb{B}^{n}$. We fix $z \in \mathbb{B}^{n}$ and let $w=t z$, where $t$ is a scalar. Then

$$
\frac{|f(z)-f(w)|}{|z-w|} \leq \frac{\beta(z, w)}{|z-w|}(g(z)+g(w)),
$$

for all $z \neq w$ in $\mathbb{B}^{n}$. Let $t$ approach 1 and apply Lemma 18; we obtain that $\left(1-|z|^{2}\right)|\mathscr{R} f(z)| \leq 2 g(z)$ for all $z \in \mathbb{B}^{n}$. Since $g \in L^{p(\cdot)}\left(\mathbb{B}^{n}, \mathrm{~d} v\right)$, then $\left(1-|\cdot|^{2}\right)|\mathscr{R} f(\cdot)| \in L^{p^{(\cdot)}}\left(\mathbb{B}^{n}, \mathrm{~d} \nu\right)$, and by Step 3 we have that $f \in A^{p(\cdot)}\left(\mathbb{B}^{n}\right)$.

In Step 7, we prove that (g) implies (a). If there exists a continuous function $g$ such that $\left(1-|\cdot|^{2}\right) g$ in $L^{p(\cdot)}\left(\mathbb{B}^{n}, \mathrm{~d} v\right)$ and for all $z, w \in \mathbb{B}^{n}$,

$$
|f(z)-f(w)| \leq|z-w|(g(z)+g(w)),
$$

then

$$
\frac{|f(z)-f(w)|}{|z-w|} \leq g(z)+g(w)
$$

Let $z=\left(z_{1}, \ldots, z_{k}, \ldots, n\right)$ and fix $z$. For each $k \in\{1, \ldots, n\}$, let $w=\left(z_{1}, \cdots, w_{k}, \ldots, z_{n}\right)$. Let $w_{k}$ tend to $z_{k}$, and we obtain

$$
\left|\frac{\partial f}{\partial z_{k}(z)}\right| \leq 2 g(z) \text {. }
$$

Therefore for all $z \in \mathbb{B}^{n}$, we obtain

$$
|\nabla f(z)| \leq 2 \sqrt{n} g(z) .
$$

Thus

$$
\left[\left(1-|z|^{2}\right)|\nabla f(z)|\right]^{p(z)} \leq\left|2 \sqrt{n}\left(1-|z|^{2}\right) g(z)\right|^{p(z)} .
$$

Since $\left(1-|\cdot|^{2}\right) g \in L^{p(\cdot)}\left(\mathbb{B}^{n}, \mathrm{~d} \nu\right)$, thus $\left(1-|\cdot|^{2}\right)|\nabla f| \epsilon$ $L^{p(\cdot)}\left(\mathbb{B}^{n}, \mathrm{~d} \nu\right)$. Therefore, we have $f \in A^{p(\cdot)}\left(\mathbb{B}^{n}\right)$ by the equivalency of (c) and (a) which we have proved.
In Step 8, we prove that (e) implies (g). If Condition (e) holds in this theorem, Lemma 1.2 in [5] says that

$$
1-\left|\varphi_{z}(w)\right|^{2}=\frac{\left(1-|z|^{2}\right)\left(1-|w|^{2}\right)}{|1-\langle z, w\rangle|^{2}} .
$$

Thus

$$
\frac{\rho(z, w)^{2}}{|z-w|^{2}}=\frac{|z-w|^{2}+|\langle z, w\rangle|^{2}-|z|^{2}|w|^{2}}{|z-w|^{2}|1-\langle z, w\rangle|^{2}} .
$$

By the Cauchy inequality, $|\langle z, w\rangle|^{2} \leq|z|^{2}|w|^{2}$, we obtain that, for all $z, w \in \mathbb{B}^{n}$,

$$
\rho(z, w) \leq \frac{|z-w|}{|1-\langle z, w\rangle|}
$$

By condition (e), there exists a continuous function $h$ in $L^{p(\cdot)}\left(\mathbb{B}^{n}, \mathrm{~d} \nu\right)$ such that, for all $z, w \in \mathbb{B}^{n}$,

$$
|f(z)-f(w)| \leq \rho(z, w)(h(z)+h(w)) .
$$

Therefore,

$$
|f(z)-f(w)| \leq \frac{|z-w|}{|1-\langle z, w\rangle|}(h(z)+h(w)) .
$$

Since

$$
\begin{aligned}
& |1-\langle z, w\rangle| \geq 1-|z| \\
& |1-\langle z, w\rangle| \geq 1-|w|
\end{aligned}
$$

we have

$$
|f(z)-f(w)| \leq|z-w|\left[\frac{h(z)}{1-|z|}+\frac{h(w)}{1-|w|}\right] .
$$

Write $g(z)=h(z) /(1-|z|)$, then

$$
|f(z)-f(w)| \leq|z-w|(g(z)+g(w)) .
$$

Since $z \in \mathbb{B}^{n}$, we have

$$
\frac{1}{1-|z|} \leq \frac{2}{1-|z|^{2}}
$$

so

$$
g(z)=\frac{h(z)}{1-|z|} \leq \frac{2 h(z)}{1-|z|^{2}}
$$

Since $h \in L^{p(\cdot)}\left(\mathbb{B}^{n}, \mathrm{~d} \nu\right)$, we have $\left(1-|\cdot|^{2}\right) g \in L^{p(\cdot)}\left(\mathbb{B}^{n}, \mathrm{~d} \nu\right)$.

\section{Data Availability}

No data were used to support this study.

\section{Conflicts of Interest}

The authors declare that they have no conflicts of interest. 


\section{Acknowledgments}

Jingshi Xu is supported by Hainan Province Natural Science Foundation of China (2018CXTD338). Rumeng Ma and Jingshi Xu are supported by the National Natural Science Foundation of China (Grant nos. 11761026 and 11761027).

\section{References}

[1] S. Bergmann, "Über die Kernfunktion eines Bereiches und ihr Verhalten am Rande. I," Journal Für Die Reine Und Angewandte Mathematik, vol. 169, pp. 1-42, 1933 (German).

[2] S. Bergman, The Kernel Function and Conformal Mapping, American Mathematical Society, New York, NY, USA, 1950.

[3] P. Duren and A. Schuster, "Bergman spaces," in Mathematical Surveys and Monographs, vol. 100, American Mathematical Society, USA, 2004.

[4] H. Hedenmalm, B. Korenblum, and K. Zhu, "Theory of bergman spaces," in Graduate Texts in Mathematics, vol. 199, Springer, New York, NY, USA, 2000.

[5] K. Zhu, "Spaces of holomorphic functions in the unit ball," in Graduate Texts in Mathematics, vol. 226, Springer, New York, NY, USA, 2004.

[6] H. Wulan and K. Zhu, "Lipschitz type characterizations for Bergman spaces," Canadian Mathematical Bulletin, vol. 52, no. 4, pp. 613-626, 2009.

[7] B. Bojarski and P. Hajlasz, "Pointwise inequalities for Sobolev functions and some applications," Studia Mathematica, vol. 106, pp. 77-92, 1993.

[8] P. Hajłasz, "Sobolev spaces on an arbitrary metric space," Potential Analysis, vol. 5, no. 4, pp. 403-415, 1996.

[9] P. Hajłasz and P. Koskela, "Sobolev met Poincaré," Memoirs of the American Mathematical Society, vol. 145, no. 688, pp. 1-101, 2000.

[10] P. Koskela and E. Saksman, "Pointwise characterizations of Hardy-Sobolev functions," Mathematical Research Letters, vol. 15, no. 4, pp. 727-744, 2008.

[11] D. Yang, "New characterizations of Hajłasz-Sobolev spaces on metric spaces," Science China Mathematics, vol. 46, no. 5, pp. 675-689, 2003.

[12] G. R. Chacón and H. Rafeiro, "Variable exponent Bergman spaces," Nonlinear Analysis. Theory, Methods \& Applications. An International Multidisciplinary Journal, vol. 105, pp. 41-49, 2014.

[13] G. R. Chacón, H. Rafeiro, and J. C. Vallejo, "Carleson measures for variable exponent Bergman spaces," Complex Analysis and Operator Theory, vol. 11, no. 7, pp. 1623-1638, 2017.

[14] D. V. Cruz-Uribe and A. Fiorenza, Variable Lebesgue Spaces: Foundations and Harmonic Analysis, Birkhäuser, Basel, Switzerland, 2013.

[15] L. Diening, P. Harjulehto, P. Hästö, and M. Ruzicka, Lebesgue and Sobolev Spaces with Variable Exponents, vol. 2017 of Lecture Notes in Mathematics, Springer, Berlin, Germany, 2011.

[16] O. Kovácik and J. Rákosnk, "On spaces $L^{p(x)}$ and $W^{k, p(x), "}$ Czechoslovak Mathematical Journal, vol. 41, no. 116, pp. 592-618, 1991.

[17] H. Rafeiro and S. Samko, "Variable exponent Campanato spaces," Journal of Mathematical Sciences, vol. 172, no. 1, pp. 143164, 2011.

[18] D. Békollé, "Intégrales à poids pour le projecteur de Bergman dans la boule unité de $C^{n}$," Studia Mathematica, vol. 71, no. 3, pp. 305-323, 1982.
[19] E. M. Stein, Harmonic Analysis, Princeton University Press, Princeton, NJ, USA, 1993. 


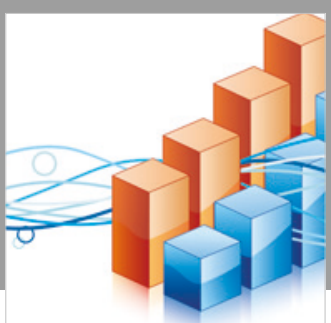

Advances in

Operations Research

\section{-n-m}
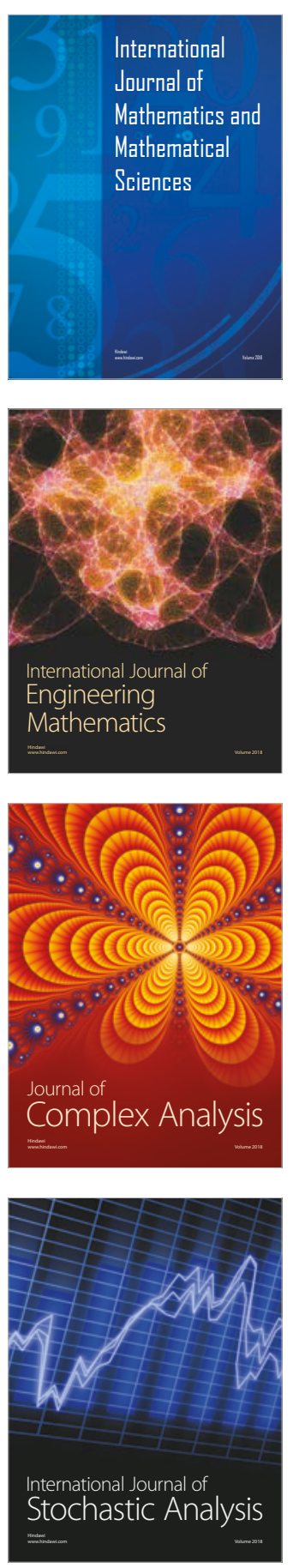
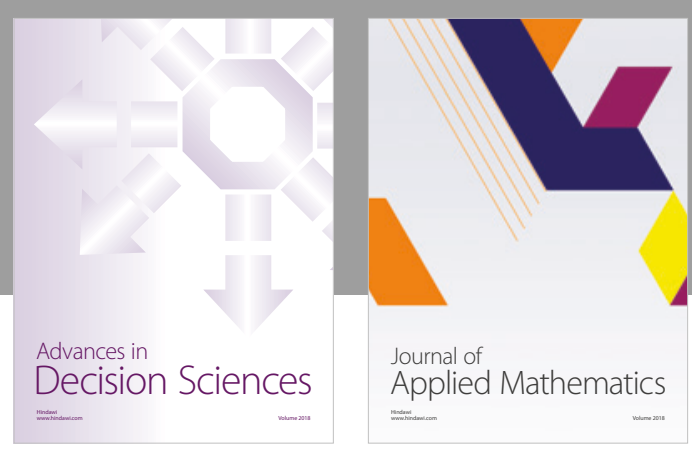

Journal of

Applied Mathematics
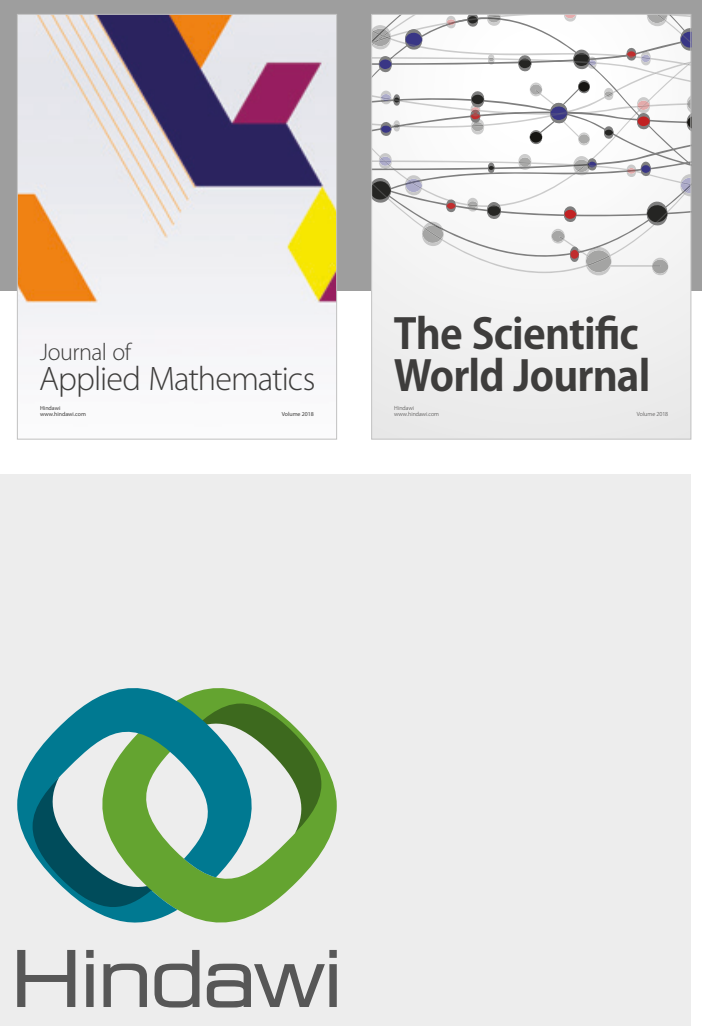

Submit your manuscripts at

www.hindawi.com

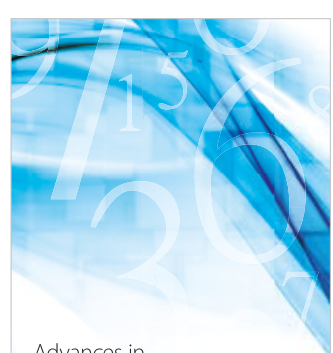

Advances in
Numerical Analysis
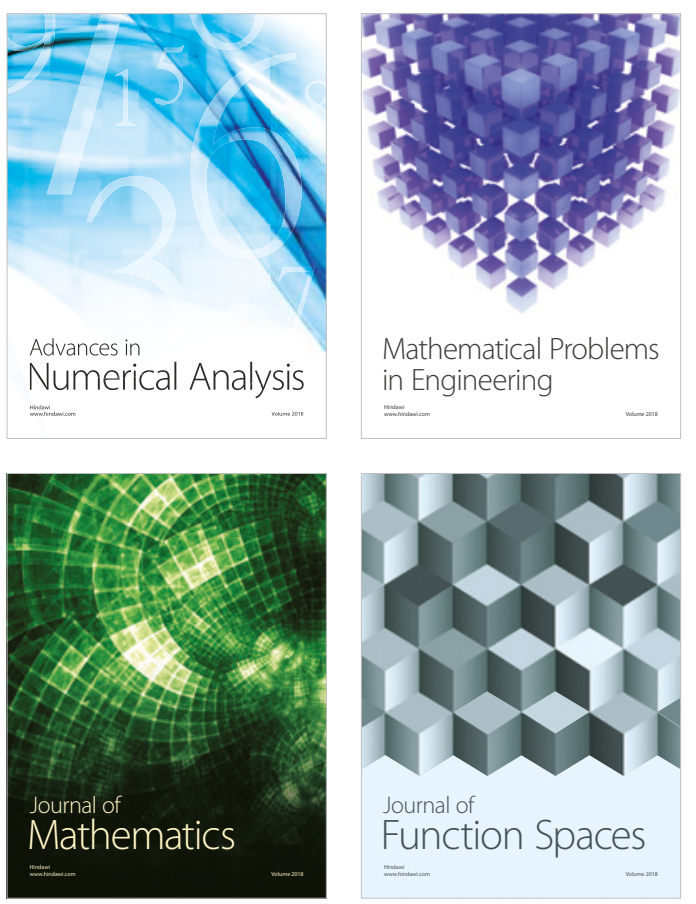

Mathematical Problems in Engineering

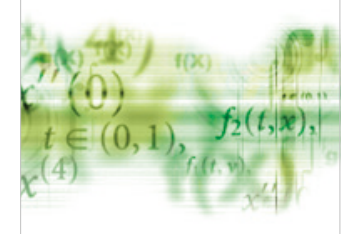

International Journal of

Differential Equations

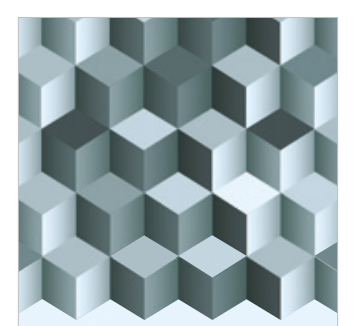

Journal of

Function Spaces

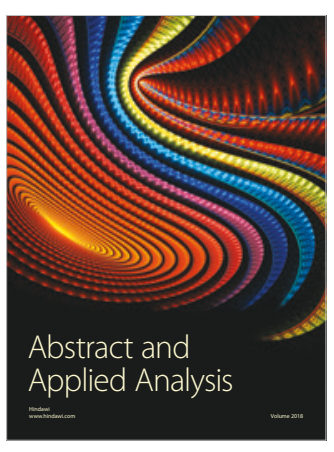

The Scientific

World Journal

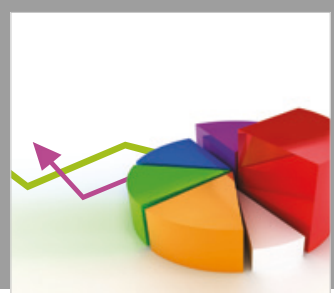

Journal of

Probability and Statistics
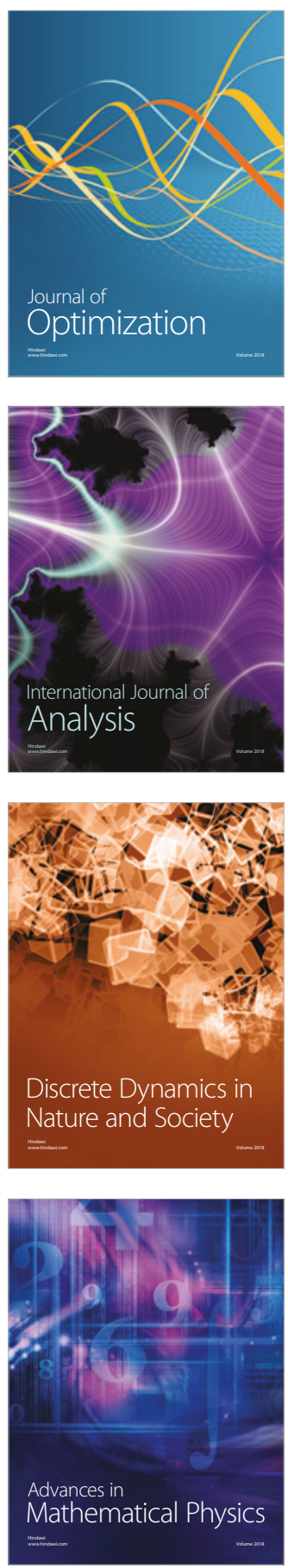